\title{
Aplicación del modelo factorial en la medición de las causas de la evasión tributaria en los comerciantes en el mercado: La Condamine, Oriental, Simón Bolívar y EMMPA, período 2019
}

Application of the factorial model in the measurement of the causes of tax evasion in merchants in the market: La Condamine, Oriental, Simón Bolivar and EMMPA, period 2019

María José Ruiz Ávila. ${ }^{1}$ \& Iván Patricio Arias González. ${ }^{2}$

DOI: $\underline{\text { https://doi.org/10.33262/visionariodigital.v5i3.1745 }}$

\begin{abstract}
.
Introduction: Tax evasion is an alien behavior and in violation of legal provisions Objective: Apply the factorial model in measuring the causes of tax evasion, know the causes that originate tax evasion and finally carry out the factorial model to measure tax evasion. tax evasion. the causes of tax evasion Methodology: the research had a qualitative approach where the information was collected through the survey, the research level was correlational that aimed to examine the relationship between the two study variables. The population of the investigation was the formal sellers of the markets of the city of Riobamba, period 2019, information taken from the GADM Riobamba cadastral base that is detailed below: Condamine Market with 741 merchants, El Prado Market with 1491 merchants, Mercado Simón Bolívar with 539 merchants and EMMPA with 899 merchants, determining a sample of 347 people Results: the main cause of tax evasion is ignorance of legal regulations Conclusion: tax evasion is very common among merchants, first due to ignorance and second, because they consider that it is not

\footnotetext{
1 Universidad Nacional de Chimborazo, Carrera de Contabilidad y Auditoría, Chimborazo, mjruiz.fpa@ unach.edu.ec, https://orcid.org/0000-0002-3075-0617

2 Universidad Nacional de Chimborazo, Carrera de Contabilidad y Auditoría, Chimborazo. ivan.arias@unach.edu.ec, https://orcid.org/0000-0002-6842-848X
} 
necessary to have a permit to be able to sell their products, which causes damage to tax control.

Keywords: Factor model, tax evasion, merchants.

\section{Resúmen}

Introducción: La evasión fiscal es una conducta ajena y violatoria a las disposiciones legales Objetivo: Aplicar el modelo factorial en la medición de las causas de la evasión tributaria, conocer las causas que originan la evasión tributaria y finalmente realizar el modelo factorial para la medición de las causas de la evasión tributaria Metodología: la investigación tuvo un enfoque cualitativo donde se recogió información mediante la encuesta, el nivel de investigación fue correlacional que tuvo como finalidad examinar la relación existente entre las dos variables de estudio. La población de la investigación fueron los vendedores formales de los mercados de la ciudad de Riobamba, período 2019, información tomada de la basa catastral del GADM Riobamba que se detalló de la siguiente manera: Mercado Condamine con 741 comerciantes, Mercado El Prado con1491 comerciantes, Mercado Simón Bolívar con de 539 comerciantes y EMMPA con 899 comerciantes, determinando una muestra de 347 personas Resultados: la principal causa de evasión tributaria es el desconocimiento de la normativa legal Conclusión: la evasión tributaria es muy común entre los comerciantes, primero es por desconocimiento y segundo porque consideran que no es necesario tener un permiso para poder vender sus productos, causando un daño al control de impuestos.

Palabras claves: Modelo factorial, evasión tributaria, comerciantes.

\section{Introducción.}

A nivel mundial los comerciantes buscan generar ganancias con la optimización de recursos, en el Ecuador, los contribuyentes de manera general evidencian un desconocimiento de la normativa tributaria (Puente \& Solano, 2020), especialmente por las constantes modificaciones realizadas por el ente regulador, en este contexto surge la evasión tributaria que representa una conducta ajena y violatoria a las disposiciones legales. Es importante señalar que el pago de impuestos en Ecuador es fundamental, porque a través de ellos se crean recursos para financiar el presupuesto general del Estado.

En los últimos años la recaudación de impuestos evidencia un incremento considerable para el Estado, con los recursos generados de la recaudación tributaria se gestionan servicios para la sociedad e impulso de la economía nacional (Párraga, 2017), sin embargo; esta acción ha sido empañada por un fenómeno recurrente como es la evasión tributaria, sobre la cual la administración tributaria lucha con normativa legal para minimizar esta práctica ilícita 
Por lo antes expuesto el objetivo de la presente investigación es aplicar el modelo factorial para identificar las causas que inciden en la evasión tributaria en los comerciantes del Mercado: La Condamine, Oriental, Simón Bolívar y EMMPA, período 2019

\section{Evasión Tributaria}

Los contribuyentes suelen realizar operaciones y transacciones financieras para minimizar sus pagos de impuestos y, por lo tanto, aumentar sus ingresos después de impuestos (Gaaya, Lakhal y Lakhal, 2017). Aunque los contadores fiscales a menudo utilizan medios legítimos para reducir o evitar las obligaciones fiscales corporativas, a veces violan el marco legal y los estándares éticos (Richardson, 2008). La evasión de impuestos consiste en acciones ilegales intencionales tomadas por empresas para reducir sus pagos y obligaciones de impuestos al declarar menos riqueza, ingresos o ventas; exagerar las deducciones y exenciones; o no declarar activos financieros (Benkraiem, 2021).

La evasión tributaria o evasión fiscal, conocida a nivel mundial como fraude fiscal es una acción ilícita, es decir, implica la violación de la ley tributaria, por lo tanto, tiene una serie de consecuencias jurídicas para sus evasores, pues implica la transgresión de la legislación tributaria vigente (Henríquez, 2015, Rodríguez, 2018). Para conseguir este propósito los contribuyentes hacen una declaración falsa o simplemente ocultan la información sobre la base del impuesto que deben declarar y el monto del tributo a pagar a la autoridad tributaria.

Los contribuyentes que son detectados practicando la evasión están sujetos a la aplicación de sanciones legales (Asamblea Nacional, 2020). Por lo tanto, practicar la evasión puede implicar un costo para el contribuyente evasor cuando éste es sorprendido a través de los procesos de fiscalización que aplica la autoridad tributaria.

Las prácticas de evasión fiscal tienen varias implicaciones sociales y económicas negativas (Culiberg \& Bajde, 2014). Debido a que el impuesto sobre la renta es un mecanismo necesario para la capacidad fiscal, la infraestructura económica y los servicios públicos y sociales proporcionados por el estado tanto en los países desarrollados como en desarrollo

Hoy en día en la ciudad de Riobamba, por parte de los comerciantes que trabajan en los mercados, se mencionan que el crecimiento del comercio aumentado en los últimos meses debido a la situación actual que atraviesa el país, lastimosamente la crisis económica ha invadido a grandes y medianos comerciantes el cual ha causado un efecto negativo al desarrollo económico de la ciudad.

Con lo antes expuesto es importante mencionar que el comercio en los mercados está relacionado con la evasión fiscal en el ámbito de la economía y finanzas; así, la tributación cobra especial importancia desde hace más de cinco décadas, sus implicancias y el grado de cumplimiento en los impuestos, consideran que la evasión de impuestos modela el proceso de decisión del cumplimiento tributario que se basa en la afirmación, que el 
contribuyente es un maximizador de ingresos personales, el emprendedor es el interesado en buscar una mayor utilidad, el problema en el cumplimiento o pago de impuestos tiene que ver con el comportamiento de los individuos, en cambio otros, cumplen con las normas y políticas tributarias, estas dos formas se relacionan a través de la moral tributaria y el cumplimiento tributario de forma directa e indirecta.

\section{Causas de la Evasión Tributaria}

Existen diferentes causas para la evasión tributaria, por la que los contribuyentes buscan la manera de evadir el pago de los tributos, que abarca varias razones a criterio del contribuyente, entre ellas están factores económicos, sociales, culturales e históricos, todos estos factores van desde las percepciones extremas hasta como ven la corrupción de un determinado manejo del estado por parte de sus gobernantes, y también de la poca satisfacción personal de los bienes y servicios recibidos por parte del estado. (Cordova, 2014)

Muchos contribuyentes por desconocer las leyes tributarias, realizan sus declaraciones de impuesto de una manera no correcta, sumándole también a esto, el deseo de evadir el pago de los tributos, así como aprovechar las debilidades que puedan tener las Administraciones Tributarias, aun sabiendo el riesgo de ser detectados. (Chelala, \& Giarrizzo, 2014).

Entre las principales causas de la evasión fiscal autores como Jorrat \& Podesta (2010) establecen la ineficacia de la fiscalización, falta de simplicidad y precisión de la normativa tributaria, carga tributaria elevada y falta de conciencia en los contribuyentes.

\section{Cultura Tributaria}

La cultura tributaria se entiende como un conjunto de valores, conocimientos y actitudes compartido por los miembros de una sociedad respecto a la tributación y la observancia de las leyes que la rigen, esto se traduce en una conducta manifestada en el cumplimiento permanente de los deberes tributarios con base en la razón, la confianza y la afirmación de los valores de ética personal (Méndez, 2004).

La cultura tributaria para autores como Alva 2010 representa cuando los individuos cumplen con los deberes formales atribuidos a este dentro de un marco legal, para cumplirlos de una manera voluntaria, conociendo que su cumplimiento acarreará un beneficio común para la sociedad en la cual ellos están insertados.

Investigaciones recientes demuestran que los atributos de la cultura tributaria son maleables y reaccionan a factores externos, en este contexto la cultura tributaria representa la responsabilidad los contribuyentes al declarar cada mes o cada seis meses de acuerdo a sus ingresos y gastos, cumpliendo según las normativas del servicio de rentas internas en el caso ecuatoriano. 


\section{Modelo Factorial}

El análisis factorial se utiliza para identificar factores que expliquen una variedad de resultados en diferentes pruebas, es una técnica estadística de reducción de datos usada para explicar las correlaciones entre las variables observadas en términos de un número menor de variables no observadas llamadas factores. Las variables observadas se modelan como combinaciones lineales de factores más expresiones de error (Sánchez, et al., 2014).

El modelo factorial es un instrumento estadístico del programa SPSS que permite conocer o detectar las factores o causas de la evasión tributaria, es muy confiable ya que arroja resultados de acuerdo al estudio de las dos variables de investigación.

\section{Metodologia.}

En la investigación se utilizó el método deductivo que ayudó al análisis de la evasión tributaria y la aplicación de un método factorial, el cual permitió determinar las incidencias en los mercados de la ciudad de Riobamba en el período 2019.

El alcance de la investigación es descriptiva, por cuanto al recolectar datos se pudo identificar y describir en forma detallada y coherente los resultados obtenidos.

La población objeto de estudio lo constituyeron los comerciantes informales de los mercados riobambeños, de acuerdo a catastro obtenido del Gobierno Autónomo Descentralizado Municipal de Riobamba como se detalla en la tabla 1.

Tabla 1 Catastro Comerciantes 2019

\begin{tabular}{lccc}
\hline \multicolumn{1}{c}{ MERCADOS } & $\begin{array}{c}\text { No. COMERCIANTES } \\
\mathbf{1 0 0 \%}\end{array}$ & Muestra & \% \\
\hline Condamine & 741 & 70 & $20.19 \%$ \\
Mercado Oriental & 1491 & 141 & $40.63 \%$ \\
Simón Bolívar & 539 & 51 & $14.69 \%$ \\
EMMPA & 899 & 85 & $24.59 \%$ \\
\multicolumn{1}{c}{ TOTAL } & $\mathbf{3 6 7 0}$ & $\mathbf{3 4 7}$ & $\mathbf{1 0 0 \%}$ \\
\hline
\end{tabular}

Nota: GADM - R (2019). Catastro Comerciantes

La técnica utilizada en la presente investigación fue la encuesta, aplicada a la población objeto de estudio a través de un cuestionario, cuyos resultados se muestran en el siguiente apartado

\section{Resultados.}

\section{Caracterización tributaria de los comerciantes}

El régimen tributario es una norma jurídica que regula las relaciones entre el Estado (sujeto activo) y quienes tienen obligaciones tributarias para con él, en este contexto en 
Ecuador existen distintos regímenes tributarios, para el año 2019 los regímenes tributarios vigentes lo constituían: régimen general y régimen impositivo simplificado, al aplicar la encuesta se obtuvo los resultados que se muestran en la tabla 2.

Tabla 2 Régimen Tributario

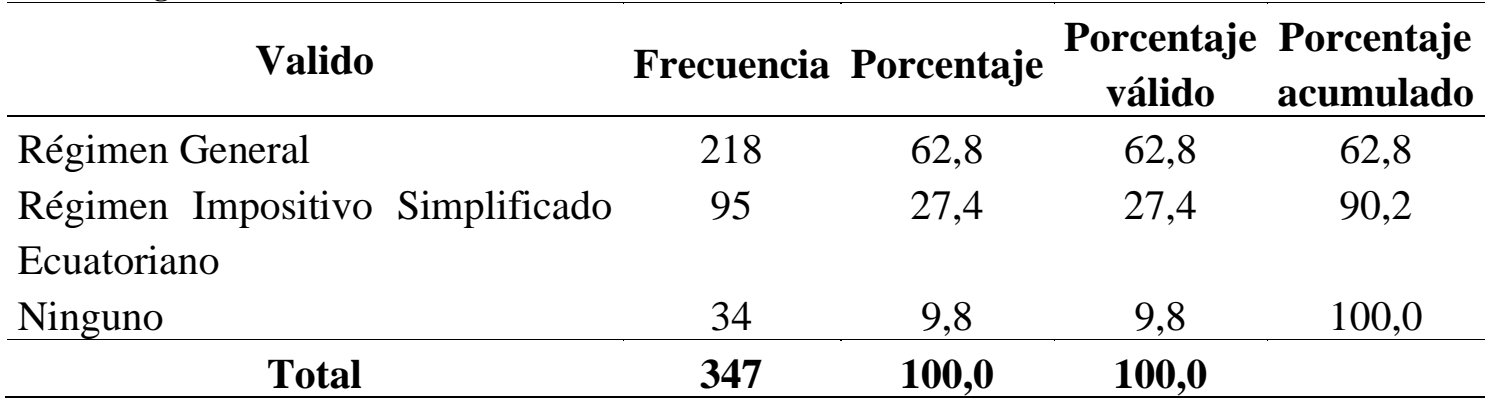

De acuerdo a los resultados obtenidos se evidencia que el 90,2\% de los comerciantes de los mercados de la ciudad de Riobamba, están vinculados a algún tipo de régimen impositivo y solamente el $9.8 \%$ no están formalizados frente a la administración tributaria.

\section{Declaración de ingresos totales}

Uno de los elementos considerados en la investigación, fue indagar qué importancia le da el comerciante a revelar el $100 \%$ de sus ventas totales frente a la administración tributaria, en este contexto se han obtenido los resultados que se muestran en la tabla 3.

\begin{tabular}{lcccc}
\hline \multicolumn{1}{c}{ valido } & Frecuencia & Porcentaje & $\begin{array}{c}\text { Porcentaje } \\
\text { Pálido }\end{array}$ & $\begin{array}{c}\text { Porcentaje } \\
\text { acumulado }\end{array}$ \\
\hline Totalmente en desacuerdo & 25 & 7,2 & 7,2 & 7,2 \\
En desacuerdo & 15 & 4,3 & 4,3 & 11,5 \\
Ni de acuerdo, ni en desacuerdo & 96 & 27,7 & 27,7 & 39,2 \\
De acuerdo & 75 & 21,6 & 21,6 & 60,8 \\
Totalmente de acuerdo & 136 & 39,2 & 39,2 & 100,0 \\
\hline \multicolumn{1}{c}{ Total } & $\mathbf{3 4 7}$ & $\mathbf{1 0 0 , 0}$ & $\mathbf{1 0 0 , 0}$ & \\
\hline
\end{tabular}

\section{Causas de la evasión tributaria en los comerciantes}

En la investigación se plantearon varias interrogantes que se resumen en las siguientes estadísticas como se muestra en la tabla 3.

Tabla 3 Estadísticas Descriptivas

\begin{tabular}{lccr}
\hline & Media & $\begin{array}{c}\text { Desviación } \\
\text { estándar }\end{array}$ & N de análisis \\
\hline $\begin{array}{l}\text { P1. ¿Está de acuerdo con los impuestos que se } \\
\text { debería pagar al SRI por su actividad }\end{array}$ & 3,22 & 1,170 & 347 \\
$\begin{array}{l}\text { comercial? }\end{array}$ & & &
\end{tabular}


P2. ¿Considera que es oportuno declarar los ingresos y gastos generados cada mes?

P3. ¿Al momento de declarar sus ingresos, considera que es importante informar el total de sus ventas?

P4. ¿Considera que es importante llevar un registro de control tanto de ingresos como de gastos?

P5. ¿Cree que es importante cumplir a tiempo con las obligaciones tributarias de acuerdo al régimen al que pertenece ya sea RUC o RISE?

P6. ¿Considera que evadir impuestos implique una penalidad?

P7. ¿Considera que es importante recibir notificaciones por parte del Servicio de Rentas Internas?

P8. ¿Considera correcto el pago de algún monto por no cumplir a tiempo sus obligaciones tributarias?

P9. ¿Considera que es correcto declarar a tiempo y así evitar sanciones?

$\begin{array}{lll}3,31 & 1,198 & 347 \\ 3,81 & 1,208 & 347 \\ 3,52 & 1,300 & 347 \\ 3,52 & 1,081 & 347 \\ 2,35 & 1,452 & 347 \\ 3,94 & 1,081 & 347 \\ 2,31 & 1,237 & 347 \\ 2,88 & 1,230 & 347\end{array}$

\section{Prueba de KMO y Bartlett.}

Este tipo de pruebas permite contrastar las correlaciones parciales entre variables

Tabla 4 Prueba de KMO y Bartlett

\begin{tabular}{|c|c|c|}
\hline \multicolumn{2}{|c|}{ Medida Kaiser - Meyer - Olkin de adecuación de muestreo } & 0,893 \\
\hline \multirow{3}{*}{ Prueba de esfericidad de Bartlett } & Aproximación Chi-cuadrado & 6708,241 \\
\hline & Grados de libertad & 36 \\
\hline & Significancia & 0,000 \\
\hline
\end{tabular}

En este contexto existe un alto grado de correlación entre las variables del análisis factorial. Para el modelo factorial se utilizó 9 preguntas en base a las dos variables de estudio, los cuales detectaron 3 causas de la evasión tributaria.

\section{Comunalidades}

Las comunalidades representan una proporción de variabilidad de cada variable, que es explicada a través de factores, en la tabla 5 se muestran los resultados obtenidos de la aplicación de encuestas a los comerciantes de los mercados de la ciudad de Riobamba en el año 2019.

Tabla 5 Comunalidades Evasión Tributaria

\begin{tabular}{lcc}
\hline \multicolumn{1}{c}{ Preguntas } & Inicial & Extracción \\
\hline $\begin{array}{l}\text { P1. ¿Está de acuerdo con los impuestos que se debería pagar al SRI } \\
\text { por su actividad comercial? }\end{array}$ & 1,000 & $\mathbf{0 , 9 5 4}$ \\
\hline
\end{tabular}


P2. ¿Considera que es oportuno declarar los ingresos y gastos generados cada mes?

$1,000 \quad 0,950$

P3. ¿Al momento de declarar sus ingresos, considera que es importante informar el total de sus ventas?

P4. ¿Considera que es importante llevar un registro de control tanto de ingresos como de gastos?

$1,000 \quad 0,933$

P5. ¿Cree que es importante cumplir a tiempo con las obligaciones tributarias de acuerdo al régimen al que pertenece ya sea RUC $\quad 1,000 \quad 0,899$ o RISE?

P6. ¿Considera que evadir impuestos implique una penalidad? $\quad 1,000 \quad 0,830$

P7. ¿Considera que es importante recibir notificaciones por parte $\begin{array}{ll}1,000 & 0,896\end{array}$ del Servicio de Rentas Internas?

P8. ¿Considera correcto el pago de algún monto por no cumplir a tiempo sus obligaciones tributarias?

P9. ¿Considera que es correcto declarar a tiempo y así evitar sanciones?

\section{Discusión}

Para realizar la comprobación de la hipótesis de investigación se utilizó la prueba estadística del Chi - cuadrado, el cual es un método para probar las hipótesis relacionadas con la diferencia entre el conjunto de frecuencias observadas en una muestra y el conjunto de frecuencias teóricas y esperadas de la misma muestra.

\section{Prueba de hipótesis general.}

H1: El desconocimiento de leyes, la falta de interés y por el alto cobro de impuestos son las causas para la evasión tributaria en los comerciantes del mercado. La Condamine Oriental, Simón Bolívar y EMMPA, periodo 2019.

$H_{0}$ : El desconocimiento de leyes, la falta de interés y por el alto cobro de impuestos no son las causas para la evasión tributaria en los comerciantes del mercado La Condamine Oriental, Simón Bolívar y EMMPA, periodo 2019.

Tabla 6 Cálculo de la aplicación Chi - cuadrado

\begin{tabular}{cccccc}
\hline $\mathbf{N}$ & $\boldsymbol{f o}$ & $\boldsymbol{f e}$ & $\boldsymbol{f o}-\boldsymbol{f e}$ & $(\boldsymbol{f o}-\boldsymbol{f e})^{\mathbf{2}}$ & $\frac{(\boldsymbol{f o - f e})^{\mathbf{2}}}{\boldsymbol{f e}}$ \\
\hline 1 & 35 & 52,44 & $-17,44$ & 304,31 & 5,80 \\
2 & 46 & 50,33 & $-4,33$ & 18,78 & 0,37 \\
3 & 130 & 92,33 & 37,67 & 1418,78 & 15,37 \\
4 & 80 & 76,67 & 3,33 & 11,11 & 0,14 \\
5 & 56 & 75,22 & $-19,22$ & 369,49 & 4,91 \\
6 & 32 & 52,44 & $-20,44$ & 417,98 & 7,97 \\
7 & 49 & 50,33 & $-1,33$ & 1,78 & 0,04 \\
8 & 110 & 92,33 & 17,67 & 312,11 & 3,38 \\
9 & 90 & 76,67 & 13,33 & 177,78 & 2,32 \\
10 & 66 & 75,22 & $-9,22$ & 85,05 & 1,13 \\
11 & 25 & 52,44 & $-27,44$ & 753,20 & 14,36 \\
12 & 15 & 50,33 & $-35,33$ & 1248,44 & 24,80 \\
13 & 96 & 92,33 & 3,67 & 13,44 & 0,15 \\
14 & 75 & 76,67 & $-1,67$ & 2,78 & 0,04 \\
15 & 136 & 75,22 & 60,78 & 3693,94 & 49,11 \\
\hline
\end{tabular}


Vol. 5, N³, p. 29-40, julio-septiembre, 2021

\begin{tabular}{|c|c|c|c|c|c|}
\hline 16 & 35 & 52,44 & $-17,44$ & 304,31 & 5,80 \\
\hline 17 & 45 & 50,33 & $-5,33$ & 28,44 & 0,57 \\
\hline 18 & 71 & 92,33 & $-21,33$ & 455,11 & 4,93 \\
\hline 19 & 97 & 76,67 & 20,33 & 413,44 & 5,39 \\
\hline 20 & 99 & 75,22 & 23,78 & 565,38 & 7,52 \\
\hline 21 & 22 & 52,44 & $-30,44$ & 926,86 & 17,67 \\
\hline 22 & 16 & 50,33 & $-34,33$ & 1178,78 & 23,42 \\
\hline 23 & 146 & 92,33 & 53,67 & 2880,11 & 31,19 \\
\hline 24 & 86 & 76,67 & 9,33 & 87,11 & 1,14 \\
\hline 25 & 77 & 75,22 & 1,78 & 3,16 & 0,04 \\
\hline 26 & 136 & 52,44 & 83,56 & 6981,53 & 133,12 \\
\hline 27 & 93 & 50,33 & 42,67 & 1820,44 & 36,17 \\
\hline 28 & 25 & 92,33 & $-67,33$ & 4533,78 & 49,10 \\
\hline 29 & 45 & 76,67 & $-31,67$ & 1002,78 & 13,08 \\
\hline 30 & 48 & 75,22 & $-27,22$ & 741,05 & 9,85 \\
\hline 31 & 12 & 52,44 & $-40,44$ & 1635,75 & 31,19 \\
\hline 32 & 25 & 50,33 & $-25,33$ & 641,78 & 12,75 \\
\hline 33 & 67 & 92,33 & $-25,33$ & 641,78 & 6,95 \\
\hline 34 & 112 & 76,67 & 35,33 & 1248,44 & 16,28 \\
\hline 35 & 131 & 75,22 & 55,78 & 3111,16 & 41,36 \\
\hline 36 & 106 & 52,44 & 53,56 & 2868,20 & 54,69 \\
\hline 37 & 119 & 50,33 & 68,67 & 4715,11 & 93,68 \\
\hline 38 & 64 & 92,33 & $-28,33$ & 802,78 & 8,69 \\
\hline 39 & 26 & 76,67 & $-50,67$ & 2567,11 & 33,48 \\
\hline 40 & 32 & 75,22 & $-43,22$ & 1868,16 & 24,84 \\
\hline 41 & 69 & 52,44 & 16,56 & 274,09 & 5,23 \\
\hline 42 & 45 & 50,33 & $-5,33$ & 28,44 & 0,57 \\
\hline 43 & 122 & 92,33 & 29,67 & 880,11 & 9,53 \\
\hline 44 & 79 & 76,67 & 2,33 & 5,44 & 0,07 \\
\hline 45 & 32 & 75,22 & $-43,22$ & 1868,16 & 24,84 \\
\hline$\Sigma$ & & & & & 833,03 \\
\hline
\end{tabular}

Nota: Elaboración en base al cuestionario aplicado a comerciantes de los mercados de la ciudad de Riobamba (2020).

Análisis: El Chi cuadrado teórico es de: $X i^{2}{ }_{c}=833,03$. El Chi cuadrado de la tabla es $X i^{2}{ }_{t}=46,1942$, es decir $X i^{2}{ }_{c}>X i^{2}{ }_{t}$, finalmente 833,03 $>46,1942$.

Interpretación: Como el $X i^{2}{ }_{c}=833,03$ es mayor a el $X i^{2}{ }_{t}=46,1942$, entonces se rechaza la hipótesis nula (Ho) y se acepta la hipótesis de investigación (Hi). El desconocimiento de leyes, la falta de interés y por el alto cobro de impuestos son las causas para la evasión tributaria en los comerciantes del mercado. La Condamine Oriental, Simón Bolívar y EMMPA, periodo 2019.

\section{Conclusiones}

- Las principales causas que arrojo el modelo factorial fue el desconocimiento de leyes con un porcentaje de varianza del $0,905 \%$, la falta de interés y el alto cobro de impuestos con un porcentaje de varianza del 90,242\% son las causas de la evasión tributaria en los comerciantes en el Mercado, La Condamine, Oriental, Simón Bolívar y EMMPA, período 2019, donde mencionan que la utilidad de las ventas es mínima y no es muy significativa la misma que se utiliza para poder 
invertir en más mercadería y no para pagar impuestos al SRI, tomando en cuenta que están conscientes de ciertas leyes tributarias pero si carecen de otras también.

- Se pudo determinar que el grado de causalidad entre la conciencia tributaria y la evasión tributaria en los comerciantes en el Mercado, La Condamine, Oriental, Simón Bolívar y EMMPA, período 2019 es de 90.24\% es un valor significativo donde los comerciantes saben de sus obligaciones tributarias, pero prefieren evadir aduciendo que no tienen tiempo, hay mucho que desconocen de las normativas y reglamentos del servicio de rentas internas.

- Los comerciantes consideran que pagar otro tipo de impuestos, además del tributario no representa ningún beneficio para ellos, además que las multas e intereses son muy altos, el mismo que va en contra de la economía de cada uno, mencionando que la utilidad de las ventas es mínima, la cual ayuda para el sustento de sus hogares y en algo poder invertir en más mercadería.

\section{Referencias bibliográficas}

Asamblea Nacional (2020). Código Tributario,

Benkraiem, R., Uyar, A., Kilic, M., \& Schneider, F. (2021). Ethical behavior, auditing strength, and tax evasion: A worldwide perspective. Journal of International Accounting, Auditing and Taxation, 100380.

Córdova, R. C. L. (2014). La evasión tributaria y su consecuencia en el desarrollo económico de país. In Crescendo, 5(2), 253-266.

Culiberg, B., \& Bajde, D. (2014). Do you need a receipt? Exploring consumer participation in consumption tax evasion as an ethical dilemma. Journal of business ethics, 124(2), 271-282.

Gaaya, S., Lakhal, N., \& Lakhal, F. (2017). Does family ownership reduce corporate tax avoidance? The moderating effect of audit quality. Managerial Auditing Journal.

GADM-R. (2019). Catastro Comerciantes Mercados

Henríquez, J. Y. (2015). Evasión tributaria: atentado a la equidad. Revista de estudios tributarios, (13), ág-171.

Jorratt y Podestá. (2010). La tributación directa, evasión en el impuesto a la renta y desafíos, capítulo IV, en Evasión y equidad en América Latina, Documento de Proyecto, Naciones Unidas, CEPAL. Chile.

Méndez, M. (2004). Cultura tributaria vs. Constitución. Espacio Abierto, 13(1), 123-137.

Parraga Pereira, D. A. (2017). Análisis de la evasión tributaria en ecuador a través de compras a empresas fantasmas; procesos de control para su detección (Bachelor's thesis). 
Puente, M., \& Solano, K.. (2020). Efectos del impuesto a la salida de divisas en el sector industrial importador de Riobamba, Ecuador (Effects of the Exit of Foreign Currency Tax in the Importing Industrial Sector of Riobamba, Ecuador). RANRevista Academia \& Negocios, 5(2).

Richardson, G. (2008). The relationship between culture and tax evasion across countries: Additional evidence and extensions. Journal of International Accounting, Auditing and Taxation, 17(2), 67-78.

Rodríguez, G. V. (2018). La evasión tributaria y sus efectos en los recursos directamente recaudados de la Municipalidad Distrital de Parobamba, 2016.

Sánchez, A., Bes, M., \& González \& Martínez, M. (2014). Análisis factorial. In Bioestadística amigable (pp. 487-512). Elsevier España.

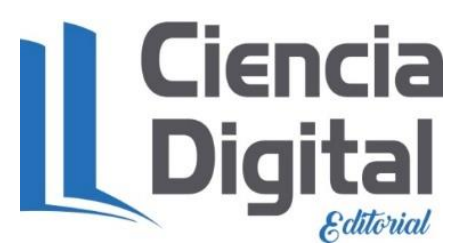




\section{PARA CITAR EL ARTÍCULO INDEXADO.}

Ruiz Ávila, M. J., \& Arias González , I. P. (2021). Aplicación del modelo factorial en la medición de las causas de la evasión tributaria en los comerciantes en el mercado: La Condamine, Oriental, Simón Bolívar y EMMPA, período 2019. Visionario Digital, 5(3), 29-40. https://doi.org/10.33262/visionariodigital.v5i3.1745

\section{\Ciencia}

El artículo que se publica es de exclusiva responsabilidad de los autores y no necesariamente reflejan el pensamiento de la Revista Visionario Digital.

El artículo queda en propiedad de la revista y, por tanto, su publicación parcial y/o total en otro medio tiene que ser autorizado por el director de la Revista Visionario Digital.
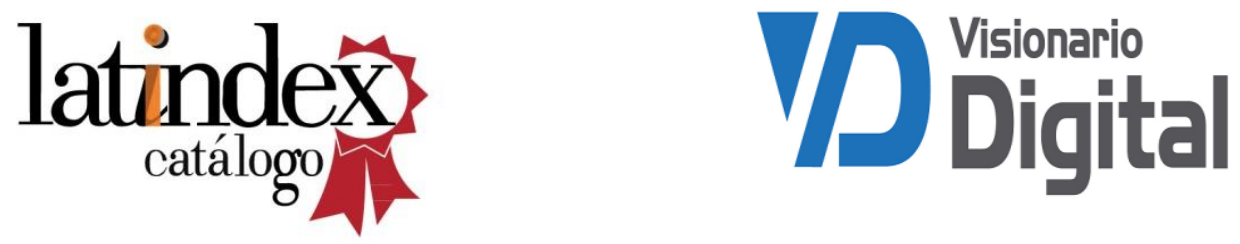